\title{
Routing based Congestion Control Metric: RFR
}

\author{
Sandeep Dalal, Ph.D \\ Asst.Professor \\ DCSA, MDU Rohtak
}

\author{
Renu Ruhil \\ M.Tech Scholar \\ DCSA, MDU Rohtak
}

\begin{abstract}
Mobile adhoc network is self configurable and adaptive. Due to node mobility we cannot predict load on the network which leads to congestion, one of the widely researched area in manets. A lot of congestion control techniques and metrics have been proposed to overcome it before its occurrence or after it has occurred. In this survey we identify the currently used congestion control metrics. Through this survey we also propose a congestion control metric RFR(resource free ratio) which considers the three most important parameters to provide congestion free route discovery. Further we show the results of node selection based on fuzzy logic calculations using the proposed metric.
\end{abstract}

\section{General Terms}

Adhoc, networks, bandwidth, buffer, adaptive.

\section{Keywords}

Congestion, manets, metric, RFR, fuzzy logic, Survey.

\section{INTRODUCTION}

Mobile adhoc network is a collection of mobile nodes which are self organize able and configure itself on the fly. These networks for their infrastructure less, quick to deploy environment provide applications in diverse domains. Since their inception they have been deployed in military areas, emergency and rescue operations, business applications and many more just because they are economical. In these networks, a mobile terminal has dual functions of a node and routing. Routes are generally broken due to node movements, load on the network, insufficient bandwidth, power insufficiency, etc. Also wireless links are unreliable and error prone. All these factors lead to congestion in the networks which jams the network and poorly affects its performance. Routing algorithms should adapt to such dynamic environment and provide quality of service and error free delivery. Congestion and load balancing are challenging tasks due to unpredictable nature of these networks.

Congestion control [18] concerns controlling traffic entry into a telecommunications network, so as to avoid congestive collapse by attempting to avoid oversubscription of any of the processing or link capabilities of the intermediate nodes and networks and taking resource reducing steps, such as reducing the rate of sending packets. It should not be confused with flow control, which prevents the sender from overwhelming the receiver. Congestion control is a key issue in mobile adhoc networks. In a network with limited shared resources and bandwidth, it is necessary to adjust the data rate used by each user in order not to overload the network. To address these issues in manets many approaches have been proposed in the literature. There are two approaches used efficiently to control routing in manets, the standard TCP with supportability for wireless networks and other through routing protocols.
In this paper we give an overview of the existing proposals and metrics, their key ideas and show their interrelation. Section 2 provides all the related work done in this field. Section 3 gives brief of the new proposed metrics. Section 4 provides the mat lab results and section 5 concludes the paper.

\section{RELATED WORK}

In recent years many researchers have tried to improve the congestion control mechanisms. The main goal of any congestion control algorithm is to balance the traffic to maximize throughput, packet delivery ratio and minimize end to end delay and packet drops.

TCP is the most popular end-to-end protocol which offers reliable connection and effective congestion control by adjusting the window size, in wired networks. A look at all the approaches proposing improved transport layer shows two major trends [1]: on the one hand many protocols try to achieve improvement while maintaining compatibility with TCP. On the other hand many approaches willingly sacrifice compatibility to gain more freedom and better fit the specific needs of manets.

In wireless network variants of TCP like TCP TAHOE, TCP RENO, NEW-RENO, VEGAS etc are used to control congestion in manets. In his paper M.M.Morshed [2] has simulated the above four TCP variants, and shows that TCP Vegas outperforms the other three TCP variants, showing the highest efficiency.

In paper [3], AODV-Multipath protocol is proposed, which selects a route from source to destination based on the queue length and hop count to avoid congestion in the network. If the queue length crosses a certain threshold value, then alternate paths are used to balance the load.

W.R.Salem and S.Hariharan in [12] focuses on congestion avoidance in terms of window size and data rate. Through simulations they conclude that Window based congestion control can resolve congestion efficiently and has higher average throughput than slow start.

In [11], A comparative study of AODV and OLSR for multiple traffic in manets with TCP congestion control have been done. Random waypoint mobility model has been used for different number of nodes for sending multiple traffic in the network. They found that number of nodes affects the performance of the network and AODV outperforms OLSR.

In [17], the authors have proposed two congestion-aware metrics, BOR and SFSR, to enable routing protocols to consider congestion issues. They have used these metrics to modify DSR to improve throughput of networks. Buffer Occupancy Ratio (BOR) indicates the utilization of the resource available in a node and its ability of packet forward packet. BOR =Occupied buffer size/buffer size. Successful Frame Sending Ratio (SFSR) indicates contention of an area and cost for sending packets through this area.

SFSR=Number of received ACKs/number of sent frames. 
In the proposed scheme once the route is selected, it will not be changes until it is broken.

In [4], a new routing protocol (EDAODV) early detection congestion and control routing protocol for wireless adhoc networks has been presented. It detects congestion by calculating queue status value and finds congestion status (CS) of a node. Based on CS, the non congested successor and predecessor nodes of a congested node are used to find alternate non congested path bi-directionally between them for data transmission. Simulations study show that EDAODV lost fewer packets than AODV that are not having congestion control mechanism.

T.Senthil Kumaran et al presents EDCSCAODV, an early detection of congestion and self cure routing protocol for wireless adhoc networks [5]. This proposed algorithm cures congestion by using local path concept, by periodically calculating the queue status of path nodes, thus detecting congestion likely to happen and send warning message and apply automatic cure algorithm to get alternate path for it. EDCSCAODV improves performance by reducing delay, routing overhead and increasing packet delivery ratio without incurring any significant cost.

In paper [7] a novel method for dynamic congestion detection and control routing (DCDR) based on the estimation of the average queue length at the node level has been proposed. This scheme is based on Congestion Free Set (CFS) to identify a congestion free path to the destination. The drawback of this algorithm is that during heaviest traffic flows it suffers from packet loss and also did not consider the wireless losses.

Bambang et. Al [16], clearly defines the transfer reliability and congestion control in opportunistic networks, which are a class of mobile adhoc networks. Open research issues have been identified.

A Mobile agent based congestion control[8] AODV protocol is proposed, in which mobile agents collect and update routing information and congestion status of the nodes using the Total Congestion Metric(TMC), thus giving dynamic network topology to nodes.

In [9], Yuang Yi et al have proposed a Hop by hop congestion control algorithm using optimization based framework, with channel access time imposed as a MAC constraint. Using the Lyapunov-function based approach they show that their algorithm is globally stable in the absence of delay. While in the presence of the delay, they proved that the algorithm has the property of spreading through simulations. They have derived bounds on the peak load at a node by hop-by hop count and end-to-end delay, proving hop-by hop scheme to have significant gains.

In [10], CBCC (Cluster Based Congestion Control) consisting of scalable and distributed cluster based mechanism for supporting congestion control in manets have been proposed. This scheme is based on the self organization of the network into clusters. Within its localized scope the clusters autonomously and proactively monitor congestion.

In paper [13], study of coding and congestion aware routing protocols have been presented. This concludes that network coding help exploit unique opportunities offered in the adhoc networks.

Survey of congestion control techniques under manets has been provided by Hitesh Gupta et.al [14], along with a novel proposed approach to control congestion in AOMDV protocol, using queue technique.
In [19], a novel AODV (AODV+SBA) routing protocol has been proposed which improves performance by reducing excessive routing overhead, by using the CFT(Congestion Free Time) metric used in MAC layer.

In survey paper[15], overview of different congestion control algorithms in manets has been presented. This paper concludes that no single congestion control mechanism is helpful; we need to combine them for efficient results.

\section{PROPOSED WORK}

In this paper we propose a new metric RFR (Resource Free Ratio) for congestion control. For effective load balancing the metrics must capture the load effectively on the network. RFR takes into account the three most important parameters for improving the network performance and reducing congestion in the network. In our approach if a nodes dynamic buffer size, bandwidth and the remaining power is below a defined threshold, then during route discovery such nodes are avoided. In this way not only the node but the congested area is discarded and hence the load is balanced equally on the network.

Dynamic buffer size $=($ current occupied buffer/allowed buffer range)

We use the mechanism suggested by [22] to calculate the available bandwidth. For the calculation of the bandwidth the idle period of the wireless channel is important.

The available bandwidth can be calculated using "Eq. (1)"

$B W_{a v}=B W_{\max }\left(\right.$ idle $_{t} /$ int $\left._{t}\right)$

Where $\mathrm{BW}_{\max }$ is the maximum available bandwidth and idle is $_{\mathrm{t}}$ the idle period of the wireless channel over the time interval int $_{\mathrm{t}}$, which can be calculated using "Eq. (2)"

idle $_{t}=$ int $_{t}-$ busy $_{t}$.

The carrier sense mechanism of IEEE 802.11 can judge whether the wireless channel is idle or busy and can be used to monitor the transition state. The above equations (1) and (2) can be used to calculate the bandwidth of the wireless link.

Estimation of battery power: The battery power [22] consumed by a node after time t can be calculated using "Eq. (3)"

$E_{c}(t)=\sigma^{*} N_{T}+\rho * N_{R}$

Where $\mathrm{N}_{\mathrm{T}}=$ number of data packets transmitted by the node after time $t ; N_{R}=$ number of data packets received after time t; $\rho, \sigma$ are constants having values between 0 and 1 . If $E_{1}$ is the initial power of the node, then the remaining power of the node $\mathrm{E}_{\mathrm{R}}$ at time $\mathrm{t}$ can be calculated using "Eq.(4)"

$E_{R}(t)=E_{c}(t)-E_{l}$ 


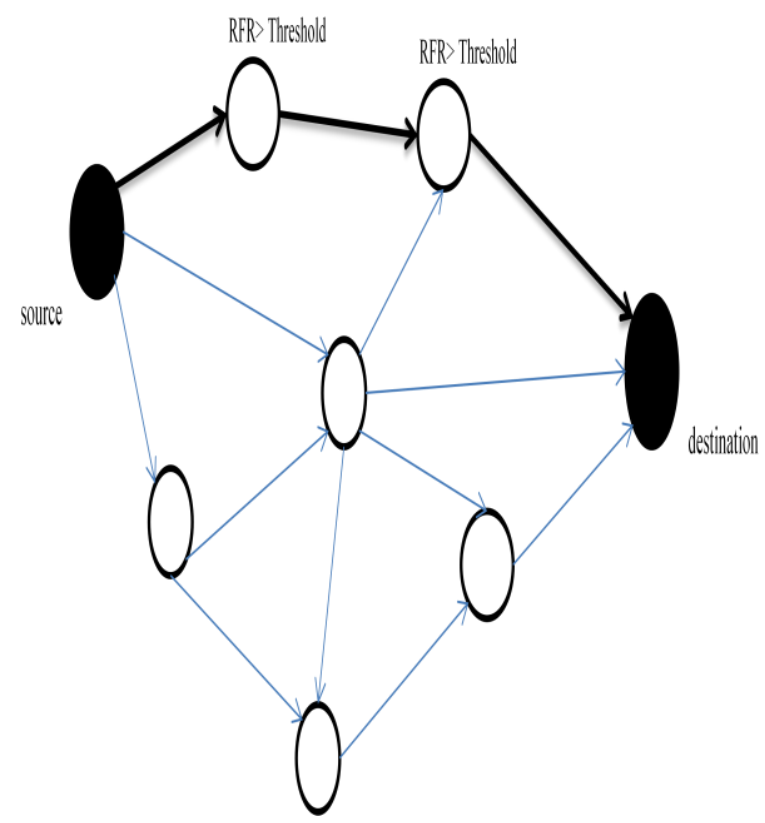

Fig 1: Route discovery using RFR

\subsection{Calculation of RFR}

During calculation of RFR all the three parameters ratio must be above the threshold value to select the node for communication.

Initially estimate Dynamic buffer size

If DBS>threshold

Next estimate available bandwidth

If $\mathrm{BW}>$ threshold,

Then finally calculate the battery power.

If $\mathrm{BP}>$ threshold,

Now RFR=Avg $(\mathrm{DBS}+\mathrm{BW}+\mathrm{BP})$

If RFR> threshold

Then select the node

Else drop the node

This metric will find the least congested node and balances the load throughout the network equally.

\subsection{Proposed Alogrithm}

During route discovery phase

1. Initiate RREQ packet by setting RFR

2. Broadcast the RREQ packet

3. At intermediate node

If $(\mathrm{RFR}<$ threshold $)$

Drop node

Else If (any intermediate or destination node has upto-date route) Else

Send RREP packet to source

Update RREQ packet and rebroadcast it to its neighbors.

This metric will find the least congested node and balances the load throughout the network equally.

\section{RESULTS USING FUZZY LOGIC}

Mat lab has been used for designing the fuzzy logic system, using which we have calculated the chances of a node to be selected or dropped. For this purpose we have coded 27 rules and taken following parameters:

1. Fuzzy Input parameter: DBS, BW, BP.

2. Fuzzy Output parameter: Probability function

According to our proposed algorithm, we have input these three parameters into the fuzzy system which produces output probability function. This output probability function gives us the probability of a node to be selected or dropped. We have taken a fixed threshold range. More the summation value of dynamic buffer size(DBS), Bandwidth(BW), Battery Power(BP) is greater than threshold range, more will be the value of probability function and hence more the chance of a node to be selected otherwise it will be dropped.

Probability $\left(\mathrm{x}_{\mathrm{i}}\right) \propto \propto \sum((\mathrm{DBS})+(\mathrm{BW})+(\mathrm{BP})) \mathrm{x}_{\mathrm{i}}$

The above equation clearly shows the probability of a node (x) is directly proportional to the summation value of three parameters. Fuzzy logic system named by metric proposed for congestion control that is RFR (Resource Free Ratio) is shown in Fig.2. Here three fuzzy variables are input and the required output probability is produced.

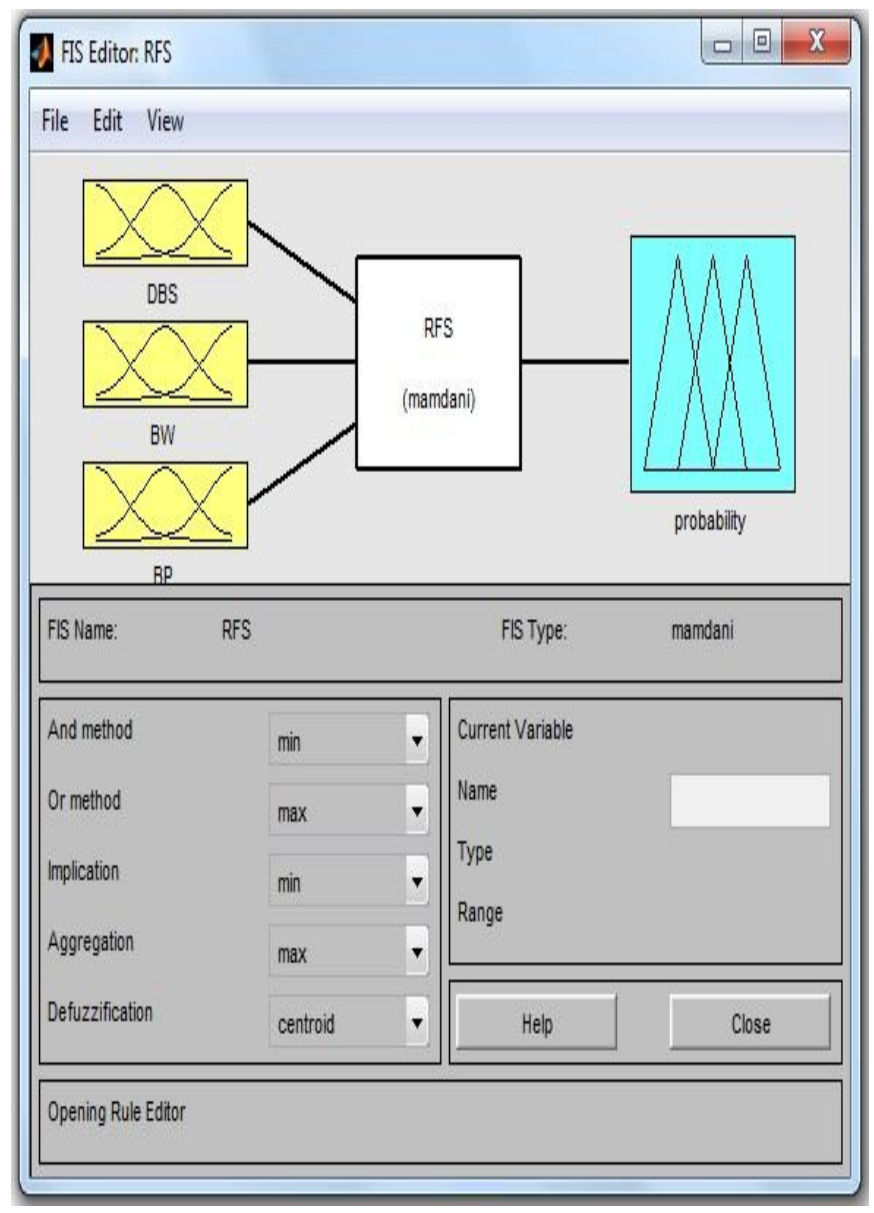

Fig 2: Fuzzy logic system

Membership function of Dynamic Buffer Size (DBS) is shown in Figure 3. Here membership function plot are shown corresponding to threshold range. 


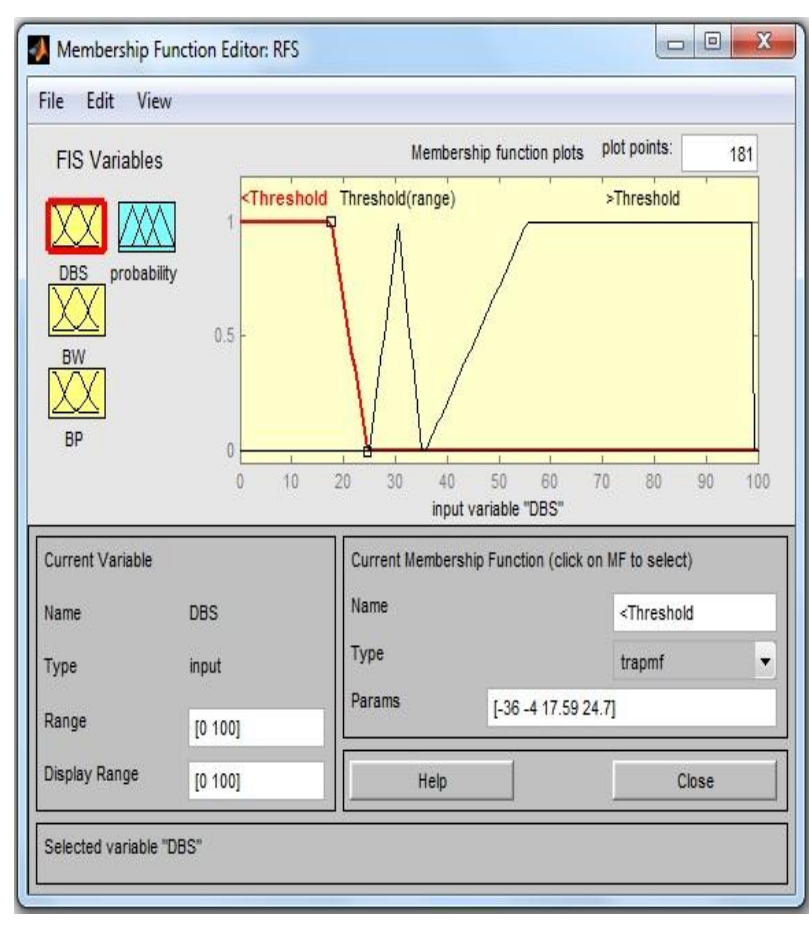

Fig 3: Membership function of DBS variable

Output probability function curve is divided into various regions namely VSmall, Small, RSmall, Medium, RHigh, High, VHigh are shown with the help of figure 4.

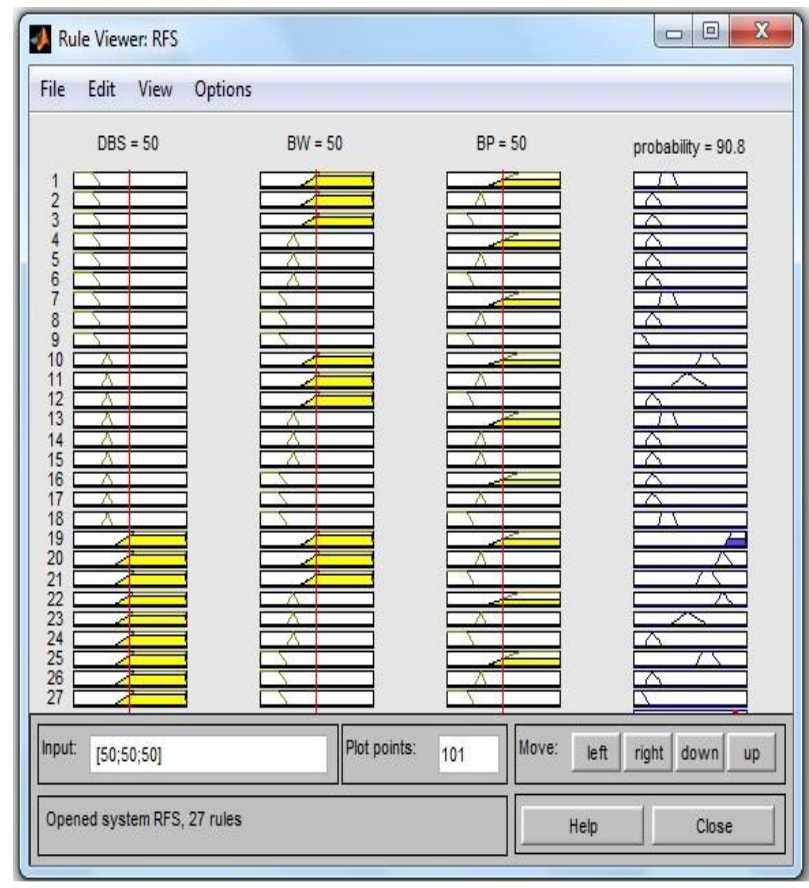

Fig 4: Fuzzy rule view

Relationship between Bandwidth (BW), Battery Power(BP) and Probability output is also shown in surface viewer in figure 5. Here $\mathrm{X}$ axis corresponds to Bandwidth (BW), $\mathrm{Y}$ axis corresponds to Battery power (BP)and $\mathrm{Z}$ axis corresponds to probability of node to be selected or dropped.

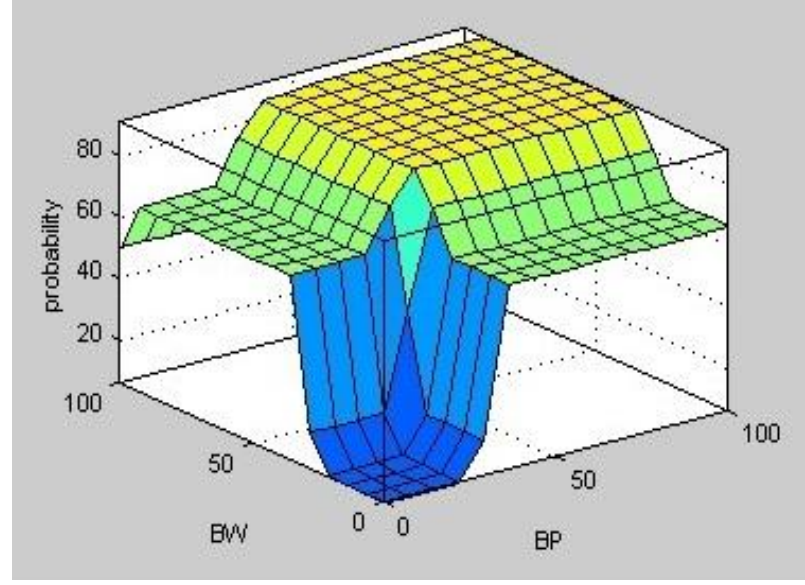

\section{CONCLUSION}

In this paper we have given an overview of almost all congestion control techniques used in mobile adhoc networks. Congestion in adhoc networks can be controlled either through routing or using the standard TCP congestion control mechanisms used in wired networks with modification and compatibility for wireless networks. Due to high node mobility and topology changes, the TCP control techniques applied to adhoc networks are inadequate to handle congestion. The congestion control metrics have been studied in detail. We further conclude that the available congestion control mechanisms and metrics are not sufficient and do not reduce packet loss up to a substantial level. Here we have proposed a metric RFR which works on the three important parameters to discover a congestion free route and balances the load on the network.

\section{Future Work}

This paper presents a congestion control metric RFR, based on calculation of available dynamic buffer size, bandwidth and battery power. In future we will be implementing this metric using NS2 in AODV and OLSR routing protocols and do their comparative study.

\section{REFERENCES}

[1] Christian Lochert, B. Scheuermann, M. Mauve, "A Survey on Congestion Control for Mobile Ad-Hoc Networks", Wiley Wireless Communications and Mobile Computing 7 (5), pp. 655-676.

[2] Md. M. Morshed, M. Ur Rahman, Md. Rafiqul Islam, "An Empirical Study on Variants of TCP over AODV routing protocol in MANET", AIUB \& TigerHATS Research Team.

[3] Shalini Puri, Dr. Satish R.D, "Congestion Aovidance and Load Balancing in AODV- Multipath using Queue length"M, Second International Conference on Emerging Trends in Engineering and Technology, ICETET-09, 2009 IEEE.

[4] T. Senthil Kumaran, V. Sankaranarayanan, "Early Detection Congestion and Control Routing In Manet IEEE, 2010

[5] T. Senthil Kumaran, V. Sankaranarayanan, "Early Congestion Detection and Self Cure Routing in Manet", Springer- Verlag Berlin Heidelberg 2011.M

[6] M.Ali, B.G Stewart, A Shahrabi, A Vallavaraj,"Congestion Adaptive Multipath Routing For Load Balancing In Mobile Adhoc Networks", 2012International Conference on Innovationsin Information Technology(IIT), 2012 IEEE. 
[7] T.SenthilKumaran,V.Sankaranarayanan,"Dynamic Congestion detection and control routing in adhoc networks", Journal of King Saud University- Computer and Information Sciences (2013) 25, 25-34.Production and hosting by ElsvierB.V.

[8] Sharma V.K, Bhadauria S.S, "Mobile Agent based Congestion Control using AODV routing protocol technique for Mobile Adhoc-Network", DOI:10.5121/ijwmn.2012.4220

[9] Yi, Y., Shakkottai, S," Hop-by-Hop congestion control Over a Wireless Multi-Hop Network", IEEE/Acm transactions Networking, 2007.

[10] Karunakaran, Thangaraj, " A Cluster Based Congestion Control Protocol for Mobile Adhoc networks", International Journal of Information Technology and Knowledge Management, Vol. 2. No. 2, pp,471-474 (2010)

[11] Makoto Ikeda, E. Kulla, M. Hiyama, L. Barolli, M. Younas, M. Takizawa,"TCP Congestion Control in MANETS Traffic Considering Proactive and Reactive Routing Protocols", $15^{\text {th }}$ International Conference on Network-Based Information Systems, IEEE, (2012).

[12] W.R.S.Jeyaseelan, S.Hariharan,"Study on Congestion Aovidance in MANET", IJCA Special Issue on "Network Security and Cryptography", NSC, (2011).
[13] Nilesh R. Rathod, Hardik J. Patel, "An Approach on Coding and Congestion Aware Routing Mechanism in MANET", International Journal of Emerging Science and Engineering (IJESE), ISSN:2319-6378, Volume-1,Issue-3, January 2013.

[14] Hitesh Gupta, Pankaj Pandey, "Survey of Routing Base Congestion Control Techniques under MANET", IEEE International Conference on Emerging Trends in Computing, Communication and Nanotechnology (ICECCN 2013).

[15] Geetika M, Mahesh G, Umesh K. Chourasia, "A Survey on Congestion Control in MANET", International Journal of Computer Science and Information Technologies, Vol. 5 (2), 2014, 998-1001.

[16] Bambang Soelistijanto and Michael P. Howarth, "Transfer Reliability and Congestion Control in Opportunistic Networks: A Survey”, IEEE Communications Surveys and Tutorials, 2013.

[17] W. Song, S. Jiang, G. Wei, "A Congestion-Aware Multipath Routing with Cross Layer Design for Wireless Mesh Networks ", Proceedings of the $15^{\text {th }}$ Asia-Pacific Conference on Communications (APCC 2009)-157.

[18] http://en.wikipedia.org/wiki/Network_congestion.

[19] P. Wannawilai, C. Sathitwiriyawong, "AODV withs sufficient bandwidth Aware Routing Protocol", IWCMC,10, July 2,2010,Caen, France.

Table 1: Summary of Congestion Control Metrics in MANETS

\begin{tabular}{|c|c|c|c|c|}
\hline Proposal & $\begin{array}{l}\text { Congestion } \\
\text { Control } \\
\text { Strategy }\end{array}$ & Congestion Control Method & $\begin{array}{l}\text { Underlying } \\
\text { routing } \\
\text { protocol }\end{array}$ & $\begin{array}{l}\text { Performance } \\
\text { evaluation/ } \\
\text { target }\end{array}$ \\
\hline $\begin{array}{l}\text { Early detection of Congestion and } \\
\text { Control[4] }\end{array}$ & $\begin{array}{l}\text { Congestion } \\
\text { Detection }\end{array}$ & $\begin{array}{l}\text { Drops packet based on queue- } \\
\text { status }\end{array}$ & EDAODV & $\begin{array}{l}\text { Packet delivery ratio, } \\
\text { End-to-End delay }\end{array}$ \\
\hline $\begin{array}{l}\text { Early Congestion detection and } \\
\text { self cure routing[5] }\end{array}$ & $\begin{array}{l}\text { Congestion } \\
\text { Detection }\end{array}$ & $\begin{array}{l}\text { Drops packet based on } \\
\text { periodically calculated queue- } \\
\text { status }\end{array}$ & EDCSAODV & $\begin{array}{l}\text { Packet delivery ratio, } \\
\text { End-to-End delay }\end{array}$ \\
\hline $\begin{array}{lll}\text { Congestion } & \text { Adaptive } & \text { multipath } \\
\text { routing [6] } & & \end{array}$ & Load balancing & $\begin{array}{l}\text { Distributes traffic if average } \\
\text { load increases beyond defined } \\
\text { threshold and residual battery } \\
\text { energy and bandwidth decreases } \\
\text { below defined threshold }\end{array}$ & QMRB & $\begin{array}{lr}\text { Packet delivery } & \text { ratio, } \\
\text { End-to-End } & \text { delay, } \\
\text { Throughput } & \end{array}$ \\
\hline $\begin{array}{l}\text { Congestion Aware multipath } \\
\text { routing with cross layer design[17] }\end{array}$ & $\begin{array}{l}\text { Congestion } \\
\text { avoidance }\end{array}$ & $\begin{array}{l}\text { Buffer Occupancy ratio(BOR) } \\
\text { and Successful frame sending } \\
\text { rate(SFSR) checks congestion } \\
\text { status of nodes }\end{array}$ & MDSR-C & $\begin{array}{l}\text { Packet delay, packet loss } \\
\text { rate, network throughput }\end{array}$ \\
\hline $\begin{array}{l}\text { Congestion Avoidance and load } \\
\text { balancing using queue length [3] }\end{array}$ & $\begin{array}{l}\text { Congestion } \\
\text { Avoidance and } \\
\text { load balancing } \\
\end{array}$ & $\begin{array}{l}\text { Queue length and hop count are } \\
\text { used to select route }\end{array}$ & $\begin{array}{l}\text { AODV- } \\
\text { Multipath }\end{array}$ & $\begin{array}{l}\text { Packet delivery ratio, } \\
\text { throughput, average } \\
\text { delay, dropped packets }\end{array}$ \\
\hline $\begin{array}{lll}\text { Sufficient } & \text { Bandwidth } & \text { aware } \\
\text { routing [19] } & & \end{array}$ & $\begin{array}{l}\text { Congestion } \\
\text { Avoidance }\end{array}$ & $\begin{array}{l}\text { Channel free time (CFT) status } \\
\text { of nodes checked during route } \\
\text { discovery }\end{array}$ & $\mathrm{AODV}+\mathrm{SBA}$ & $\begin{array}{lr}\text { Packet delivery } & \text { ratio, } \\
\text { End-to-End } & \text { delay, } \\
\text { Normalized } & \text { Routing } \\
\text { Overhead. } & \\
\end{array}$ \\
\hline $\begin{array}{l}\text { Dynamic Congestion } \\
\text { and Control [7] }\end{array}$ & $\begin{array}{l}\text { Congestion } \\
\text { Detection and } \\
\text { Control }\end{array}$ & $\begin{array}{l}\text { Congestion Free set (CFS) } \\
\text { connects one hop and two hop } \\
\text { neighbors and identifies a } \\
\text { congestion free path }\end{array}$ & DCDR & $\begin{array}{lr}\text { Packet delivery } & \text { ratio, } \\
\text { End-to-End } & \text { delay, } \\
\text { routing overhead } & \end{array}$ \\
\hline $\begin{array}{l}\text { Mobile agent based congestion } \\
\text { control[8] }\end{array}$ & $\begin{array}{l}\text { Congestion } \\
\text { Avoidance }\end{array}$ & $\begin{array}{l}\text { Total Congestion Metric (TCM) } \\
\text { obtained using queue length and } \\
\text { channel contention }\end{array}$ & ABCC-AODV & $\begin{array}{l}\text { Packet delivery fraction, } \\
\text { throughput, average end- } \\
\text { to-end delay }\end{array}$ \\
\hline $\begin{array}{l}\text { Congestion Free Routing metric } \\
\text { (proposed) }\end{array}$ & $\begin{array}{l}\text { Congestion } \\
\text { avoidance }\end{array}$ & $\begin{array}{l}\text { Resource Free Routing (RFR) } \\
\text { considers dynamic buffer size, } \\
\text { battery power and bandwidth } \\
\text { for route selection }\end{array}$ & CFAODV & $\begin{array}{l}\text { Packet delivery ratio, } \\
\text { End-to-End delay , } \\
\text { Throughput }\end{array}$ \\
\hline
\end{tabular}

